\title{
Molecular cloning and characterization of the repetitive DNA sequences that comprise the constitutive heterochromatin of the $\mathrm{W}$ chromosomes of medaka fishes
}

\author{
Yusuke Takehana - Kiyoshi Naruse - Yusuke Asada • Yoichi Matsuda • \\ Tadasu Shin-I • Yuji Kohara • Asao Fujiyama • Satoshi Hamaguchi • \\ Mitsuru Sakaizumi
}

Published online: 29 November 2011

(C) Springer Science+Business Media B.V. 2011

\begin{abstract}
Among the medaka fishes of the genus Oryzias, most species have homomorphic sex chromosomes, while some species, such as Oryzias hubbsi and Oryzias javanicus, have heteromorphic ZW sex chromosomes. In this study, a novel family of repetitive sequence was molecularly cloned from $O$. hubbsi and characterized by chromosome in situ and filter hybridization, respectively. This repetitive element, which we designated as a Bst NI family element, localized at heterochromatin regions on the $\mathrm{W}$ chromosome, as well as on two pairs of autosomes. Homologous sequences to this element were found only in $O$. javanicus, which is a sister species of $O$.
\end{abstract}

Responsible Editors: Tariq Ezaz and Jennifer Graves.

Y. Takehana $(\bowtie) \cdot K$. Naruse

Laboratory of Bioresources,

National Institute for Basic Biology,

Okazaki 444-8585, Japan

e-mail: takehana@nibb.ac.jp

Y. Asada $\cdot$ Y. Matsuda

Laboratory of Animal Cytogenetics, Biosystems Science

Course, Graduate School of Life Science,

Hokkaido University,

Sapporo 060-0810, Japan

Y. Matsuda

Department of Biological Sciences,

Graduate School of Science, Hokkaido University,

Sapporo 060-0810, Japan hubbsi, suggesting that this repeated element originated in the common ancestor of these two species. However, the intensity of the hybridization signals was lower in O. javanicus than in $O$. hubbsi, and the chromosomal location of this element in $O$. javanicus was confined to heterochromatin regions on one pair of autosomes. Thus, we hypothesize that this repetitive element was extensively amplified in the $O$. hubbsi lineage, especially on its $\mathrm{W}$ chromosome, after the separation of the $O$. javanicus lineage. In addition, we also found the $\mathrm{W}$ chromosomal location of the 18S-28S ribosomal RNA genes in both $O$. hubbsi and O. javanicus. Our previous studies showed no linkage

T. Shin-I $\cdot$ Y. Kohara

Center for Genetic Resource Information,

National Institute of Genetics,

Mishima 411-8540, Japan

\begin{abstract}
A. Fujiyama
National Institute of Informatics,

Tokyo 101-8430, Japan
\end{abstract}

\section{S. Hamaguchi $\cdot$ M. Sakaizumi}

Institute for Science and Technology, Niigata University,

Niigata 950-2181, Japan 
homology of the sex chromosomes in these species, indicating that the RNA genes were shared between W chromosomes of different origins. This situation may be explained by a translocation of the sex-determining region with the ribosomal RNA genes in either species or an independent accumulation of the RNA genes as a convergent process during $\mathrm{W}$ chromosome degeneration.

Keywords Dmy. Oryzias - repetitive element . sex chromosome

$\begin{array}{ll}\text { Abbreviations } \\ \text { BLAST } & \text { Basic Local Alignment Search Tool } \\ \text { DAPI } & \text { 4',6-Diamidino-2-phenylindole } \\ \text { EST } & \text { Expressed sequence tag } \\ \text { FISH } & \text { Fluorescence in situ hybridization } \\ \text { FITC } & \text { Fluorescein isothiocyanate } \\ \text { LG } & \text { Linkage group } \\ \text { rDNA } & \text { Ribosomal DNA }\end{array}$

\section{Introduction}

Many vertebrates undergo genetic sex determination controlled by sex chromosomes. Mammals exhibit male heterogamety (XX females and XY males), while birds and snakes show female heterogamety ( $\mathrm{ZZ}$ males and ZW females). These sex chromosomes share similar properties; the $\mathrm{X}$ and $\mathrm{Z}$ chromosomes are large and gene-rich, whereas the $\mathrm{Y}$ and $\mathrm{W}$ chromosomes are smaller, highly heterochromatic, and contain only a small number of genes. However, recent cytogenetic mapping studies showed no homology among human, chicken, and snake sex chromosomes, suggesting different evolutionary origins of these sex chromosomes (Nanda et al. 1999; Matsubara et al. 2006). Therefore, the accumulation of heterochromatin and the gene loss on $\mathrm{Y}$ and $\mathrm{W}$ chromosomes are considered to be a convergent property of sex chromosome differentiation.

It has been hypothesized that all sex chromosomes originated from a pair of autosomes (Ohno 1967). In this theory, the first step is the occurrence of a novel sex-determining locus on an autosome, and at this stage of evolution, both sex chromosomes have similar shape (homomorphic). Heteromorphic sex chromosomes are thought to have arisen through suppression of meiotic recombination around the sexdetermining locus, maintaining the non-recombining region in a constant heterozygous state, and leading to numerous deletions of functional genes and accumulation of repetitive DNA sequences in a sex-specific chromosome (Y or W chromosome) (Charlesworth et al. 2005). Later, this degenerative process spreads the sex-specific region over almost the entire chromosome. However, most cytogenetic and genomic studies have focused on highly differentiated sex chromosomes in mammals and birds, and thus, the early process of the sex chromosome differentiation remains unclear.

Teleost fishes are a good model for studying the early evolutionary process of sex chromosome differentiation because their sex chromosomes differ in their origin and degeneration level. The medaka fishes in the genus Oryzias show extremely high diversity in sex determination systems and sex chromosomes. In this group, both XY and ZW sex chromosome systems have been identified, and their sex chromosomes are not homologous to each other (Fig. 1). These findings suggest the frequent appearance of new sex chromosomes from autosomes during evolution of Oryzias fishes, possibly in association with the formation of new sex-determining genes. Morphologically homomorphic sex chromosomes have been observed in four species, Oryzias latipes, Oryzias curvinotus, Oryzias luzonensis, and Oryzias dancena (Kondo et al. 2004; Matsuda et al. 1998; Takehana et al. 2007a; Tanaka et al. 2007). On the other hand, Oryzias hubbsi and Oryzias javanicus have heteromorphic ZW sex chromosomes with different origins (Takehana et al. 2007b, 2008).

In $O$. hubbsi, the W chromosome is larger than the $\mathrm{Z}$ chromosome, and two heterochromatin blocks are located near the telomeric region only on the $\mathrm{W}$ chromosome (Takehana et al. 2007b). In O. javanicus, a heterochromatin block is located at the centromeric region on the $\mathrm{W}$ chromosome, but not on the $\mathrm{Z}$ chromosome (Takehana et al. 2008). Differentiation of the sex chromosomes has progressed to a stage at which they have become cytogenetically distinct in these species.

In this study, we have identified and isolated a novel repetitive sequence family, designated as a $B s t \mathrm{NI}$ family, in $O$. hubbsi. We carried out its molecular cytologenetic characterization and demonstrated its accumulation at the heterochromatin 
Fig. 1 Phylogenetic relationships and sex determination mechanisms in Oryzias fishes. Phylogenetic information is based on Takehana et al. (2005)

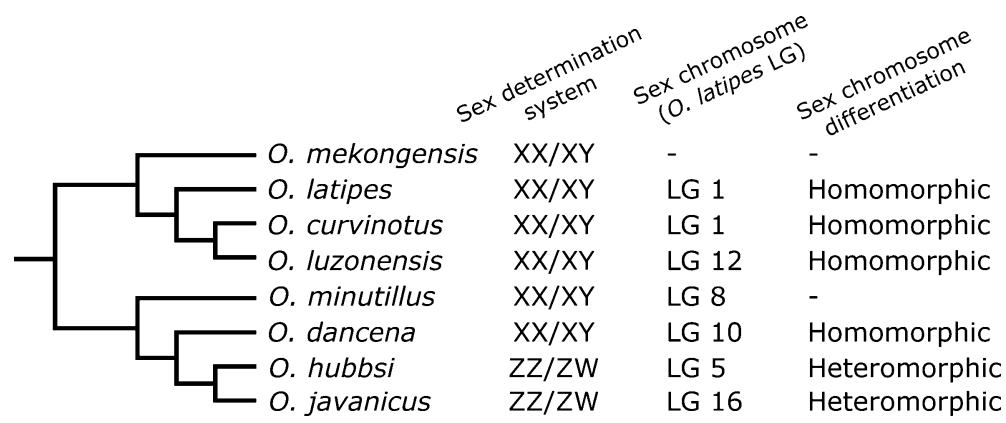

regions on the $\mathrm{W}$ chromosome, as well as on two pairs of autosomes. We also found that the $18 \mathrm{~S}-28 \mathrm{~S}$ ribosomal RNA genes were located on the W chromosomal heterochromatin of $O$. hubbsi. These results suggest that the heterochromatic regions of the W chromosome of $O$. hubbsi are composed of different types of repetitive elements. In addition, the ribosomal RNA genes were shared between the nonhomologous W chromosomes of $O$. hubbsi and $O$. javanicus, suggesting a translocation of the sexdetermining region with the RNA genes in either species or an independent accumulation of the RNA genes in both $\mathrm{W}$ chromosomes.

\section{Materials and methods}

Fish specimens

We used two males and two females of $O$. hubbsi and two females of $O$. javanicus for fluorescence in situ hybridization (FISH). The same specimens were also used in our previous studies (Takehana et al. 2007b, 2008). The original wild stocks were collected at Tangerang, Indonesia in 1992 (O. hubbsi) and Penang, Malaysia in 1995 (O. javanicus) (see Takehana et al. 2005). To examine the distribution of repetitive sequences within the genus Oryzias, genomic DNA was isolated from ten Oryzias species: Oryzias celebensis (male), O. curvinotus (male), O. dancena (male), O. hubbsi (female), O. javanicus (female), $O$. latipes (female), O. luzonensis (male), O. marmoratus (male), O. mekongensis (male), and O. minutillus (male). These fishes were supplied by the National Bio-Resource Project Medaka (NBRP Medaka) in Japan. Phenotypic sex was determined by secondary sexual characteristics of adult fish, namely, the shapes of dorsal and anal fins.
Molecular cloning of repetitive DNA and sequencing

A fosmid library with fivefold genome coverage was constructed from an $\mathrm{F}_{1}$ hybrid female between $O$. hubbsi female and O. javanicus male using the CopyControl Fosmid Library Production kit (Epicentre) to obtain genomic clones derived from the $\mathrm{W}$ chromosome of $O$. hubbsi (Takehana et al. 2007b). Fosmid clones containing sex-linked expressed sequence tags (ESTs) of $O$. hubbsi were isolated by colony hybridization. Subsequent FISH analyses using these $\mathrm{W}$ chromosomal clones demonstrated that clone F45 01 contained a sex-linked marker that hybridized to the $\mathrm{W}$ chromosomal heterochromatin regions. The clone was then digested with restriction enzymes and subcloned into pUC19 vector. The nucleotide sequences at both ends of a subclone that produced an identical FISH pattern were determined using an automated sequencer (Applied Biosystems, Japan) after a sequencing reaction with a BigDye Terminator v3.1 Cycle Sequencing Kit using M13 primers according to the manufacturer's protocol (Applied Biosystems).

The nucleotide sequence of the entire fosmid clone F45_01 was determined by the shotgun method. Briefly, the fosmid DNA was extracted using a Qiagen Large Construct Kit (Qiagen, Tokyo, Japan), following the manufacturer's protocol, and then sheared into approximately 3-kb fragments using HydroShear (Genomic Solutions, Ann Arbor, MI, USA). The end-repaired DNA fragments were fractionated by agarose gel electrophoresis and cloned into the pUC19 vector digested with SmaI (Fermentas, Vilnius, Lithuania). Shotgun reads were obtained using an ABI 3730xl genetic analyzer (Applied Biosystems). Approximately 1,500 sequence reads were collected and assembled into contigs using a parallel contig assembly program (Huang et al. 2003). Dot plot analysis of the nucleotide sequence 
was performed with Dotter (Sonnhammer and Durbin 1995).

Chromosome preparation and FISH

Chromosome preparation and FISH were performed as described previously (Matsuda and Chapman 1995; Takehana et al. 2007a, b). Chromosome slides were made from fibroblast cells taken from caudal fin tissue. FISH analysis was performed for chromosomal localization of the repetitive sequences on F45_01 and the 18S-28S rRNA genes in $O$. hubbsi and $O$. javanisus. Two cDNA clones (MF01FFA019g17 for 18S and MF01FFA031p02 for 28S) were mixed and used as probes for chromosome mapping of the 18S28S rRNA genes. These cDNA clones were supplied by the NBRP Medaka. For chromosome mapping of the sex chromosomal markers in $O$. hubbsi and $O$. javanicus, two fosmid clones, F10_01 (for O. hubbsi; Takehana et al. 2007b) and F11_01 (for O. javanicus; Takehana et al. 2008) were used as probes to identify their sex chromosomes. The probes were labeled with biotin-16-dUTP (Roche, Indianapolis, IN) or digoxigenin-11-dUTP (Roche) by nick translation, and hybridization was carried out at $37^{\circ} \mathrm{C}$. After overnight hybridization, probes were detected with avidin-FITC (Roche) and rhodamine-labeled antidigoxigenin antibodies (Roche), and then counterstained with 4',6-diamidino-2-phenylindole (DAPI). The chromosome slides were observed under an Olympus fluorescence microscope BX50 using three filters: WIB, WIG, and WU (Olympus, Tokyo, Japan). FISH images were captured with a DP72 digital camera (Olympus).

Southern blot and slot blot hybridization

Male and female genomic DNAs $(10 \mu \mathrm{g})$ of $O$. hubbsi were digested with three restriction enzymes ( $N c o$ I, $H i n f I$, and $B s t \mathrm{NI}$ ), and genomic DNAs of $O$. celebensis, O. dancena, O. hubbsi, O. javanicus, and O. minutillus were digested with HinfI. The digested DNAs were separated on a $1 \%$ agarose gel by electrophoresis, and the DNA fragments were then transferred onto nylon membranes (Hybond-N+, GE Healthcare, Buckinghamshire, UK). A mixture of 100 bp ladder plus (Fermentas, Vilnius, Lithuania) and HindIII-digested lambda DNA (NEB, Tokyo, Japan) was used as molecular size markers.
Hybridization was carried out with a repetitive sequence fragment labeled by an AlkPhos direct labeling and detection system (GE Healthcare), according to the manufacturer's instructions. Signals were detected by chemiluminescent reagents (CDPStar, GE Healthcare) and quantified using an image analyzer (LAS3000mini, Fujifilm, Tokyo, Japan).

To examine the conservation of the repetitive DNA sequence in the genus Oryzias, slot blot hybridization was performed using genomic DNA isolated from the ten Oryzias species mentioned above. The genomic DNA was denatured with $\mathrm{NaOH}$ and blotted onto nylon membranes using Bio-Dot SF blotting equipment (Bio-Rad Laboratories, Hercules, CA, USA). Hybridization and detection of the signals were carried out as described above.

\section{Results}

Molecular cloning of a repetitive sequence located on the $O$. hubbsi W chromosome

The karyotype of $O$. hubbsi comprises 23 pairs of acrocentric chromosomes and 1 pair of heteromorphic ZW sex chromosomes. DAPI-stained heterochromatin bands were previously observed at the telomeric regions on four pairs of autosomes and on the $\mathrm{W}$ chromosome (Takehana et al. 2007b). During the course of the FISH mapping study in $O$. hubbsi, we found that fosmid clone F45_01 contained a sexlinked EST that strongly hybridized to the telomeric regions on two pairs of acrocentric chromosomes (Fig. 2). The chromosomal distribution of hybridization signals and their sizes were identical to those of DAPI-stained heterochromatin on two out of the four chromosome pairs. In females, strong hybridization signals were also observed at the two DAPI-positive heterochromatin regions on the $\mathrm{W}$ chromosome, suggesting that this fosmid clone contained repetitive elements that comprised the $\mathrm{W}$ chromosomal heterochromatin.

Nucleotide sequence of the repetitive sequence element

Repeated subcloning and FISH mapping showed that a 4.3-kb fragment digested with HindIII and SacI produced the identical FISH pattern to Fig. 2 (data not 
Fig. 2 Chromosomal distributions of the repetitive DNA fragment in O. hubbsi. a and b FISH using the repetitive sequence (fosmid clone, F45_01) (red) with a sex chromosomal marker (fosmid clone, F10_01) (green) on metaphase spreads from female (a) and male (b). c and $\mathbf{d}$ Inverted DAPI pictures of the same metaphase spreads as in (a) and (b), respectively. Arrows indicate $\mathrm{Z}$ and $\mathrm{W}$ sex chromosomes. Solid and open arrowheads show heterochromatin regions with and without intense hybridization signals of the repetitive element, respectively. Scale bar represents $10 \mu \mathrm{m}$
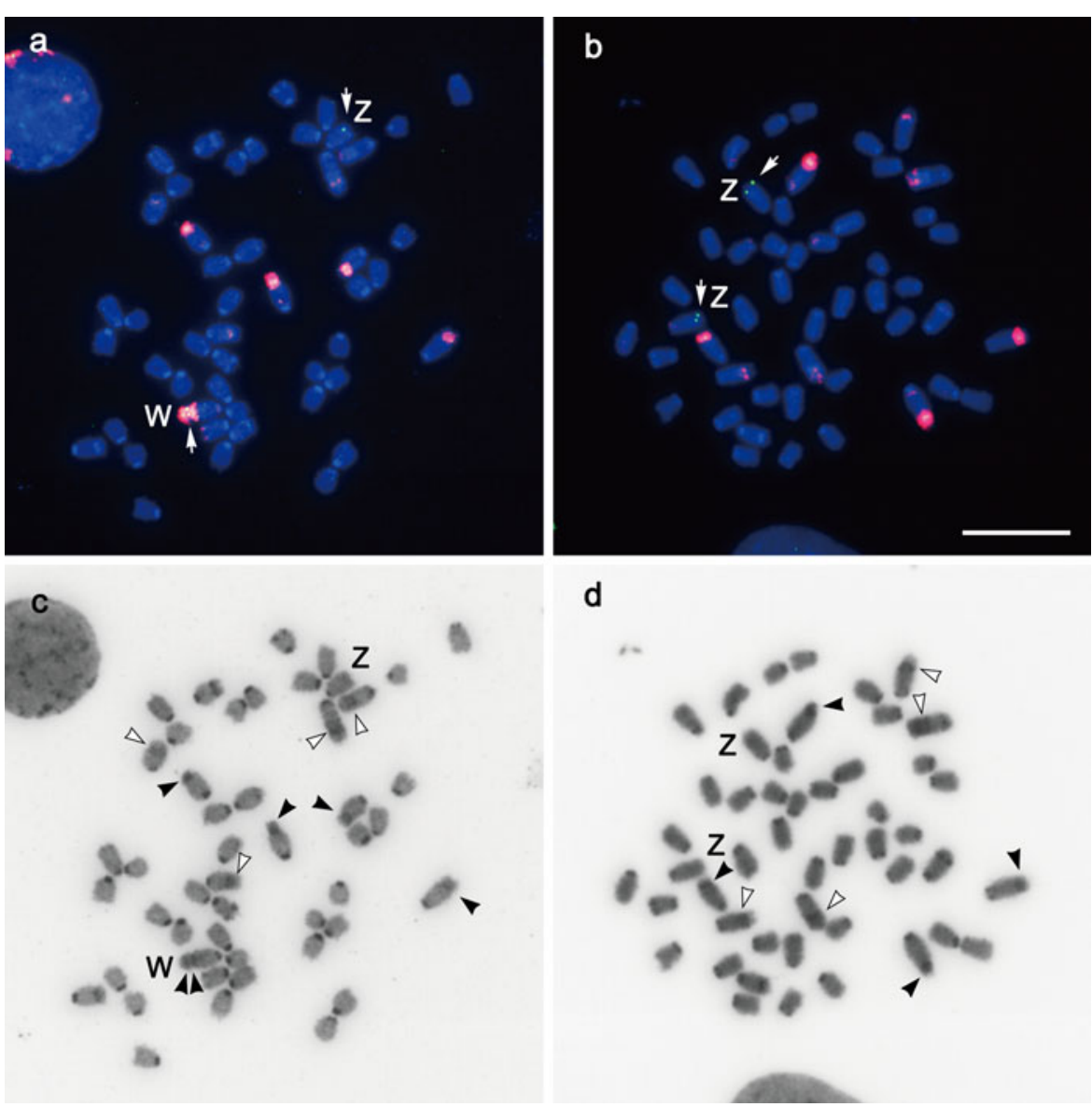

shown). Although we determined both end sequences of the $4.3-\mathrm{kb}$ clone, we could not obtain the internal sequences by primer walking because of a tandem array of repetitive elements of over $2 \mathrm{~kb}$. To further characterize the repetitive unit and its length, we then determined the entire nucleotide sequence of the fosmid clone $(35 \mathrm{~kb})$ by a shotgun method (DDBJ/ GenBank accession number: AB673114). Dot plot analysis using the complete sequence revealed the presence of internal repeats in the fosmid clone (4,332-7,259-bp region; Fig. 3a), which were included in the 4.3-kb fragment digested with HindIII and SacI (2,996-7,321-bp region). This repetitive region consisted of seven tandem repeat units with a length of 277-463 bp each (Fig. 3b, c). The GC content of this region was $37.7 \%$, indicating that this repetitive sequence was AT-rich. The nucleotide sequence was searched for homology against the NCBI database using Basic Local Alignment Search Tool (BLAST) (BLASTN and BLASTX; http://www.ncbi.nlm.nih. gov/), but no sequences with significant homology were detected. In addition, we also performed BLAST search against the RepBase ver. 14.03 (Genetic Information Research Institute, http://www.girinst. org/), which is a database specialized for repetitive sequences, while no significant homology with known repetitive sequences was found. Alignment of nucleotide sequences of the seven repetitive units showed a common Bst NI site in all units (Fig. 3c). In addition, two units (\#4 and \#6) shared an NcoI site, and six (\#1-6) shared a Hinfl site. FISH using the 463-bp fragment digested with Bst NI as a probe showed an identical hybridization pattern to that of the 4.3-kb fragment (data not shown). Therefore, we designated the repetitive sequence as a BstNI family element.

\section{Genomic organization of the repetitive sequence}

To examine the genomic organization of the Bst NI family sequences in $O$. hubbsi, male and female genomic DNA digested with three restriction enzymes 

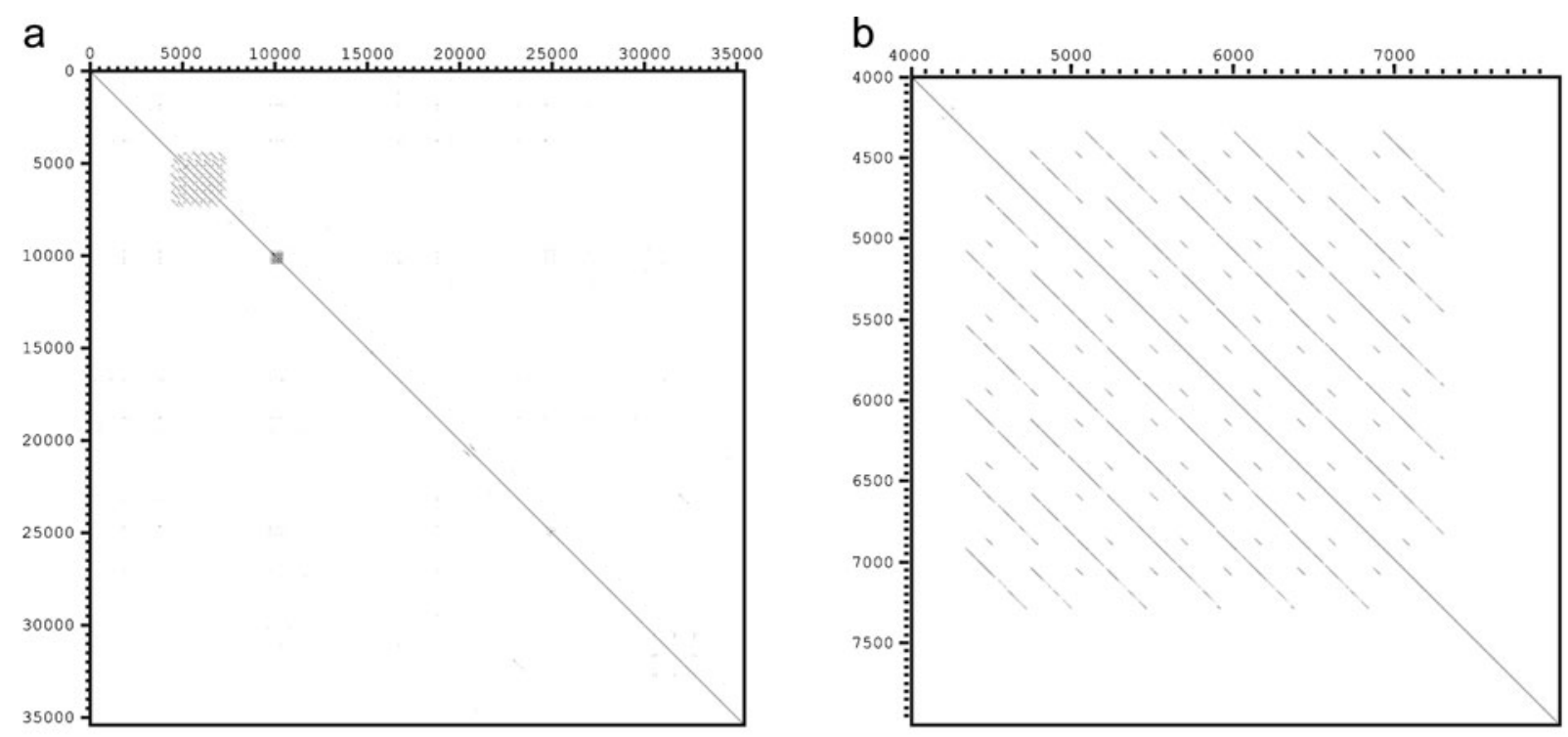

C

$\# 1$ -

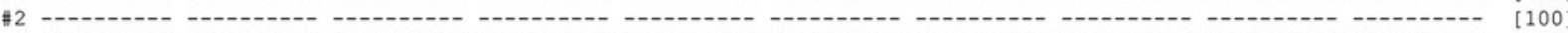

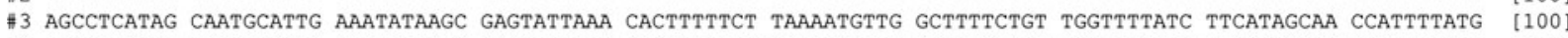

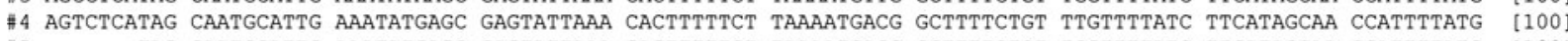
\#5-----TAG CAATGCATTG AAATATGAGC GAGTATTAAA CACTTTTTCT TAAAATGACG GCTTTTCTGT TGGTTTTATC TTCATAGCAA CCATTTTATG [100] $\# 6$------TAG CAATGCATTG AAATATGAGC GAGTATTAAA CACTTTTTCT TAAAATGACG GCTTTTCTGT TGGTTTTATC TTCATAGCAA CCATtTTATG [100] \#7 AGTCTCATAG CAATGCATTG AAATATTAGC GAGTATTAAA CACTTTTTCT TAAAATGACG GTTTTCTGT TGGTTTTATC TTCATAGCAA CCATTTTATG [100]

\#1 ------ - \#2 ----D-- -- --

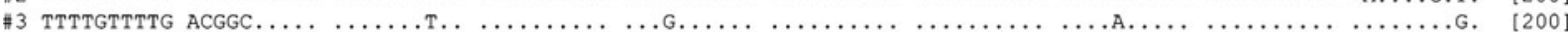

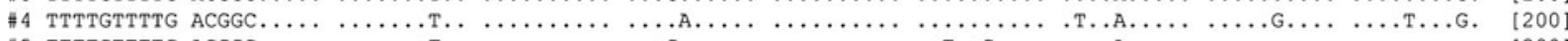

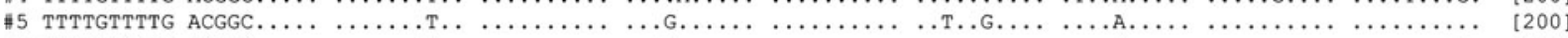

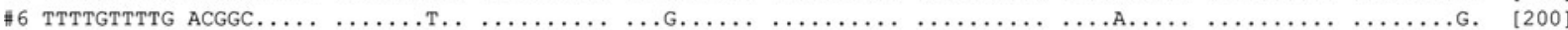

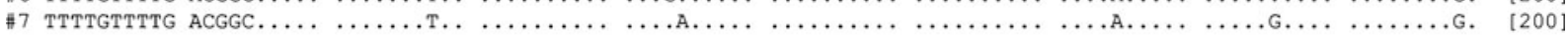

\#1 TGAATGTGA- -GAAATTGAT TAAAAAA--- GCACTTTTTT C--AAAATGG CGGCTTTTCT GTTGGTTTTA TCTCCATAGC AACCATTTTA TGTTCTGTTC [300]

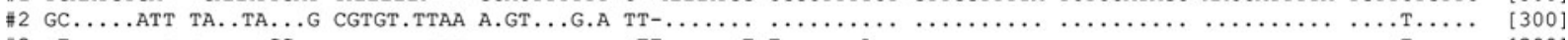

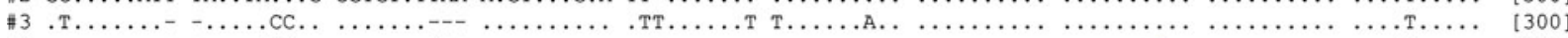

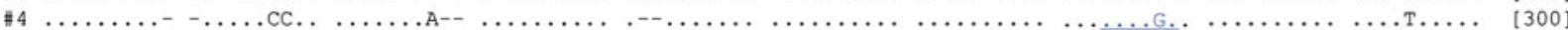

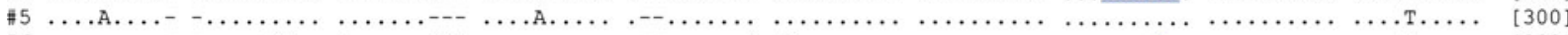

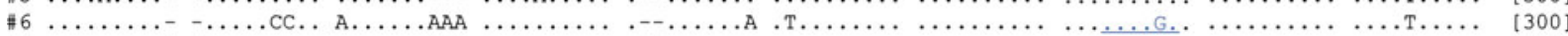

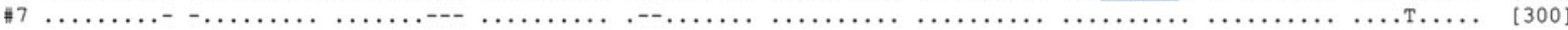

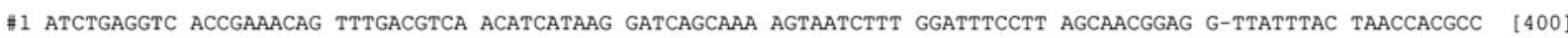

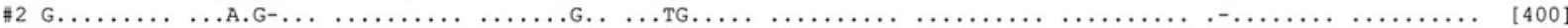

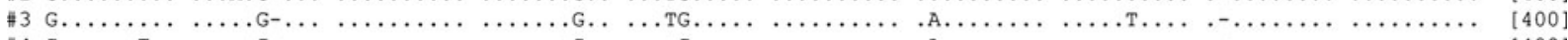

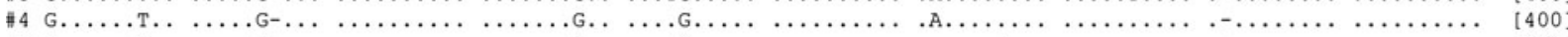

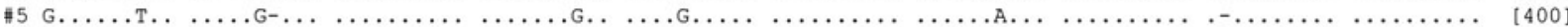

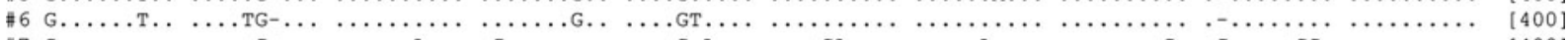

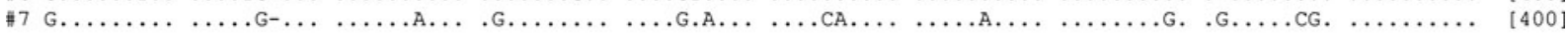

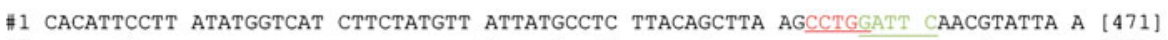

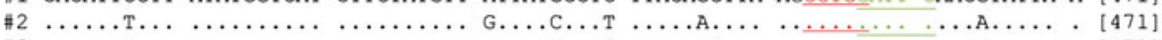

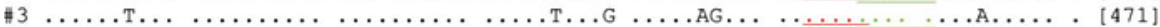

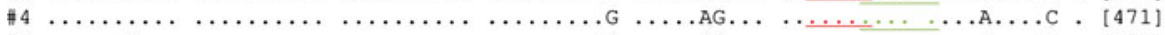

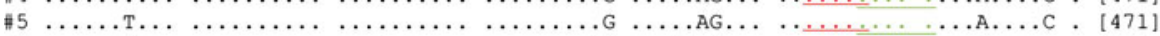

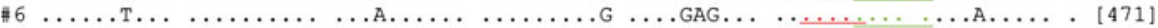

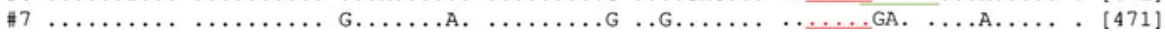

Fig. 3 Nucleotide sequence analysis of the Bst NI fragment. a Dot matrix pattern of the fosmid clone (F45_01) sequence, including the repetitive element. b High magnification of the 4,000-8,000-bp region in (a). c Alignment of Bst $\mathrm{NI}$ sequences in the fosmid clone, which comprise seven (1-7) units of an approximately $0.5-\mathrm{kb}$ element. The bases shown in blue, green, and red indicate the restriction sites of NcoI, HinfI, and Bst NI, respectively 
(NcoI, HinfI, and Bst NI) was subjected to Southern blot hybridization probed with the 463-bp BstNI fragment. The hybridization signals of the fragment were observed as polymeric ladder bands in the $B s t \mathrm{NI}$ digest that comprised the $0.5-\mathrm{kb}$ monomer unit, indicating that this sequence was tandemly arrayed in the genome of $O$. hubbsi (Fig. 4). Hybridization bands other than the polymeric ladder bands of the $B s t \mathrm{NI}$ monomer unit were probably derived from internal Bst $\mathrm{NI}$ sites in the polymeric fragments. The intensity of the hybridization signals was higher in the

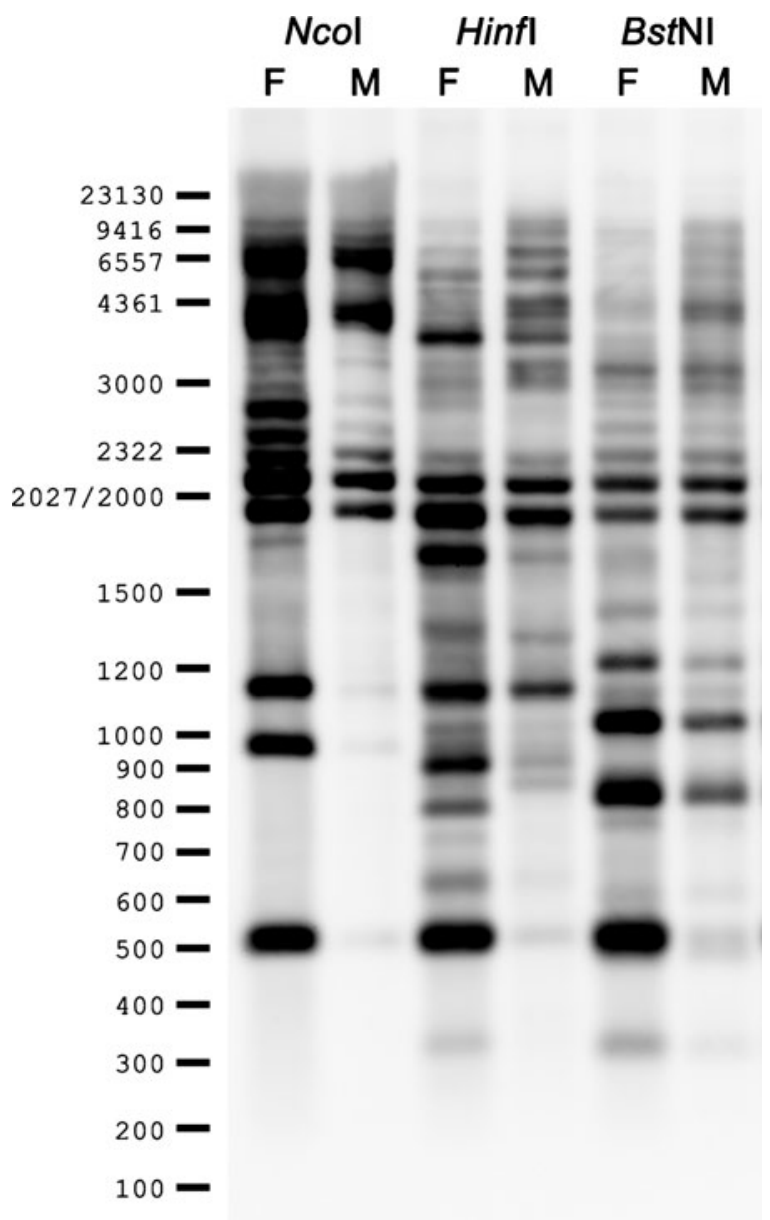

Fig. 4 Southern blot hybridization patterns of $O$. hubbsi genomic DNA probed with the 463-bp Bst NI fragment. Each lane contains $10 \mu \mathrm{g}$ of genomic DNA from male $(M)$ and female $(F)$. A mixture of $100 \mathrm{bp}$ ladder plus and HindIIIdigested lambda DNA was used as a molecular size marker female sample than in the male sample, and the 0.5 $\mathrm{kb}$ monomer band was specific to the female genome, suggesting a W chromosome-specific amplification of the $B s t \mathrm{NI}$ family monomer sequence. In addition, intensely hybridizing bands with a length of $0.5 \mathrm{~kb}$ were commonly observed in all female genomic DNAs digested with the three restriction enzymes, indicating that the restriction sites contained in the $B s t \mathrm{NI}$ repeated element were highly conserved in the female genome.

The presence of the BstNI family repetitive sequence in the genus Oryzias

To investigate the distribution of the repetitive element in the genus Oryzias, slot blot hybridization was performed for the genomic DNA of ten Oryzias species (Fig. 5a). Intense hybridization signal for the 463-bp Bst NI fragment was detected in the genomic DNA of $O$. hubbsi, while a weak signal was also detected in $O$. javanicus. The Southern hybridization signals of the fragment were observed as polymeric ladder bands in the genomic DNA of $O$. javanicus digested with HinfI, which consisted of $0.3-\mathrm{kb}$ HinfI monomer units (Fig. 5b), indicating that this sequence was tandemly arrayed in the $O$. javanicus genome. FISH using the $B s t \mathrm{NI}$ fragment as a probe was then performed for the chromosomes of O. javanicus. Intense hybridization signals were observed at the centromeric heterochromatin regions on a pair of acrocentric chromosomes having a secondary constriction, but no distinct signals were observed on the ZW sex chromosomes (Fig. 6).

Location of the $18 \mathrm{~S}-28 \mathrm{~S}$ ribosomal RNA genes on $O$. hubbsi and $O$. javanicus chromosomes

The nuclear genes encoding rRNAs [ribosomal DNAs (rDNAs)] consist of a large number of copies of a single transcription unit, clustered in long direct tandem arrays. The rDNA sequences represent useful chromosome landmarks; therefore, we also examined the chromosomal locations of the 18S-28S rDNA sequences in $O$. hubbsi and $O$. javanicus. Both species show a diploid chromosome number of $2 \mathrm{n}=$ 48 , and active nucleolar organizer regions (NORs) have been identified by silver staining at a telomeric region of an acrocentric pair in $O$. hubbsi, and at a secondary constriction on an acrocentric pair in $O$. 
Fig. 5 Distribution of the $B s t \mathrm{NI}$ repetitive element in the genus Oryzias. a Slot blot hybridization probed with the $B s t$ NI fragment. Each slot contains $500 \mathrm{ng}$ of genomic DNA. b Southern blot hybridization probed with the Bst $\mathrm{NI}$ fragment for six Oryzias species (see lane labels). Each lane contains $10 \mu \mathrm{g}$ of genomic DNA digested with HinfI. cel $O$. clebensis; cur O. curvinotus; dan O. dancena; hub $O$. hubbsi; jav O. javanicus; lat $O$. latipes; luz O. luzonensis; mar O. marmoratus; mek O. mekongensis; min $O$. minutillus a
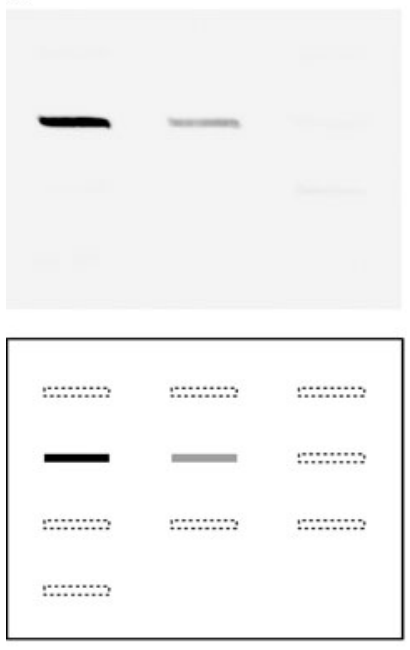

cel के cur के dan ô

hub $q$ jav $q$ lat ㅇ

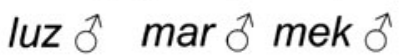
$\min \widehat{\sigma}^{\lambda}$ javanicus (Uwa and Iwata 1981; Uwa 1986). The fluorescence hybridization signals of the rDNAs appeared to localize to the same region as the b

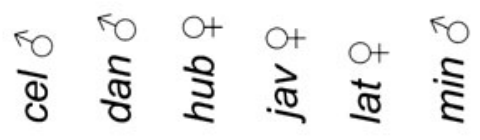

b

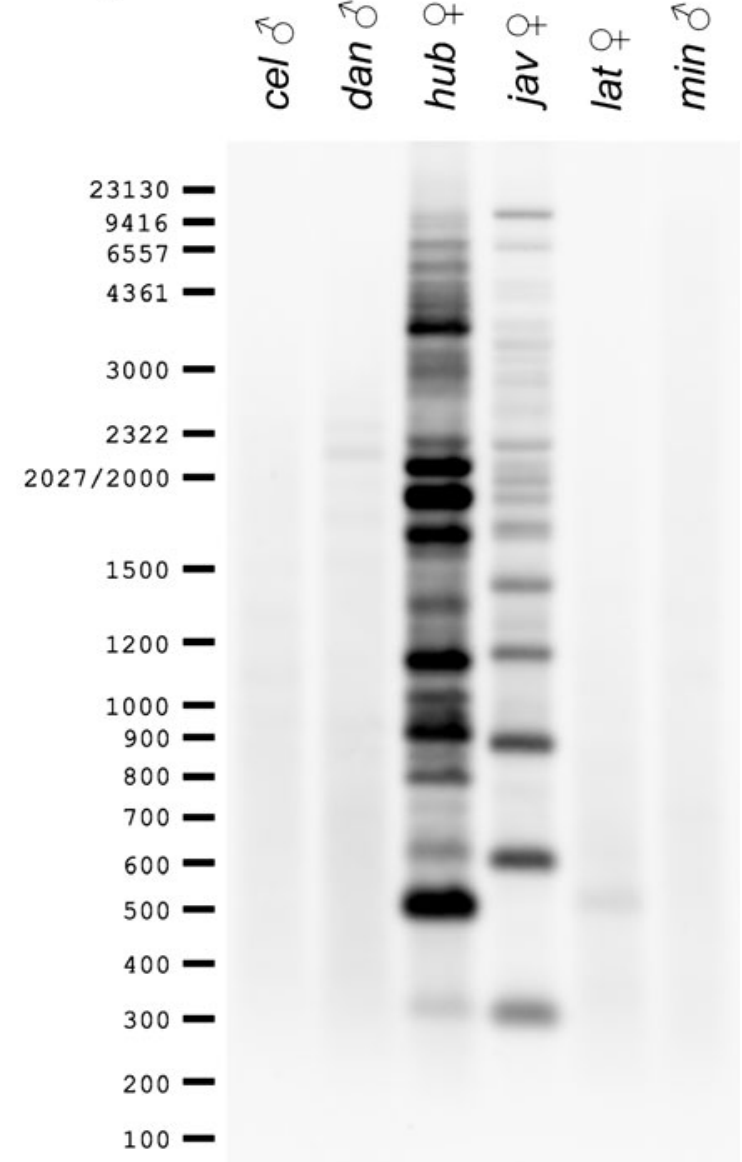

Fig. 6 FISH pattern of the $B s t \mathrm{NI}$ sequence in $O$. javanicus. a FISH using the repetitive element (red) with a sex chromosomal fosmid clone, F11_01, (green) on metaphase spreads from a female. b Inverted DAPI picture of the same metaphase spreads as in (a). Scale bar represents $10 \mu \mathrm{m}$

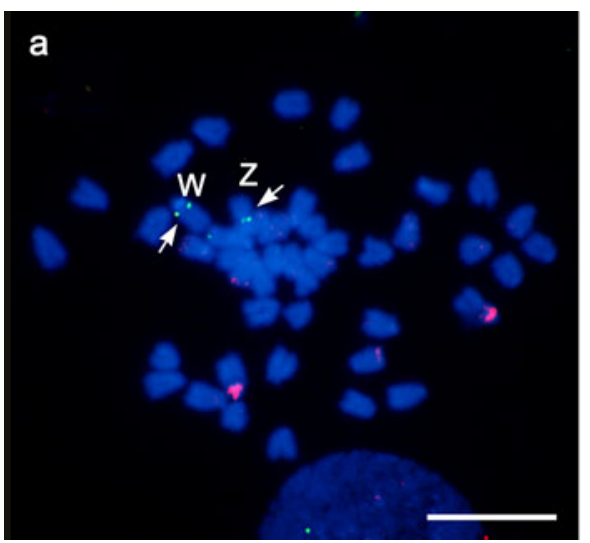

b

NOR in each species (Fig. 7). In addition, the rRNA genes were also clustered on the $\mathrm{W}$ chromosomes in both $O$. hubbsi and $O$. javanicus, which corresponded 
Fig. 7 FISH patterns of the 18S-28S rRNA genes in $O$ hubbsi and O. javanicus. a and b Chromosome locations of the rRNA genes (cDNA clones, MF01FFA019g17 and MF01FFA031p02) (red) with the sex chromosomal marker (F10_01 or F11_01) (green) on metaphase spreads from $O$. hubbsi female (a) and $O$. javanicus female (b). c and d Inverted DAPI pictures of the same metaphase spreads as in (a) and (b), respectively. Scale bar represents $10 \mu \mathrm{m}$
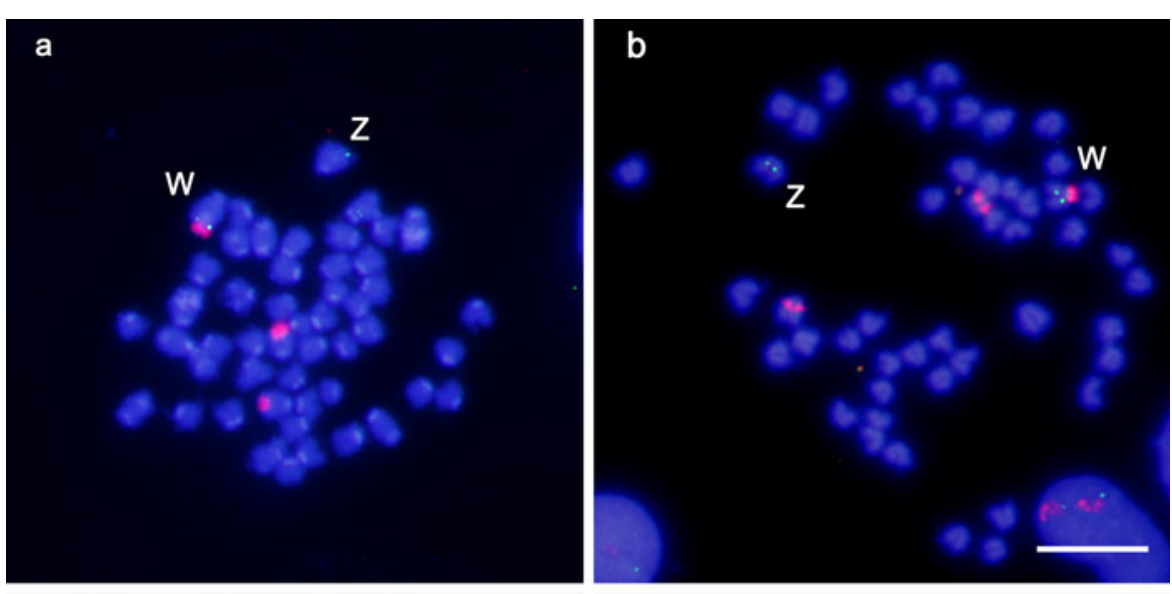

C

d

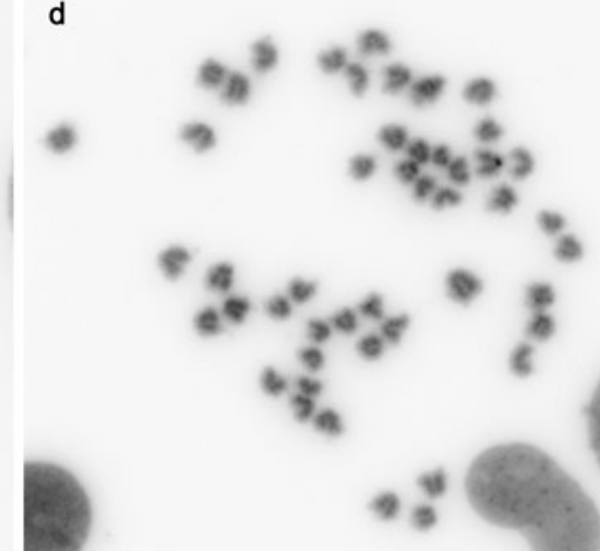

to the $\mathrm{W}$-specific heterochromatin regions (Fig. 7). The sex chromosomes of $O$. hubbsi and $O$. javanicus are homologous to different autosomes [linkage group (LG)5 and LG16] of $O$. latipes, indicating that the nonhomologous W chromosomes share the amplifications of the rDNA sequences. The rDNAs and the large clusters of the $B s t \mathrm{NI}$ repetitive element localized to same autosomes in $O$. javanicus but not in $O$. hubbsi (data not shown).

\section{Discussion}

Repeat accumulation on the W chromosome of $O$. hubbsi

O. hubbsi has heteromorphic ZW sex chromosomes, and two DAPI-positive heterochromatin blocks are located near the telomeric region on the $\mathrm{W}$ chromosome. In this study, we cloned a novel family of repetitive DNA from $O$. hubbsi, amplified specifically at the heterochromatin regions of the $\mathrm{W}$ chromosome. FISH mapping to metaphase chromosomes demonstrated that this Bst NI family repetitive element was also accumulated on heterochromatic regions of two autosomal pairs, but not on the $\mathrm{Z}$ chromosome. Furthermore, Southern hybridization revealed that this element was tandemly repeated in the genome, and that the $0.5-\mathrm{kb}$ monomer units, digested with NcoI, HinfI, and Bst NI, were specific to female genome. The GC content was low (37.7\%), and sequence homology searching using BLAST did not show the presence of any related DNA sequences. Taken together, these results suggest that this repetitive sequence is categorized as a novel satellite DNA sequence (Singer 1982) and is a good molecular cytogenetic marker for the $\mathrm{W}$ chromosome of $O$. hubbsi.

Slot blot hybridization of the repeated element to genomic DNA of ten Oryzias species revealed that nucleotide sequences with homology to the BstNI family element were rarely or not present in other 
species, with the exception of $O$. javanicus. A molecular phylogeny of Oryzias species supported a sister group relationship between $O$. hubbsi and $O$. javanicus (Takehana et al. 2005), suggesting that this repeated element originated in the common ancestor of the two species. However, the intensity of the hybridization signals was much lower in $O$. javanicus than in $O$. hubbsi. Furthermore, the chromosomal location of this element in $O$. javanicus was confined to the heterochromatin region on one pair of autosomes. Thus, we assumed that this repetitive element was extensively amplified in the $O$. hubbsi lineage, especially on its $\mathrm{W}$ chromosome, after the separation of the $O$. javanicus lineage.

In our previous study, we found that the entire length of the $\mathrm{W}$ chromosome is larger than that of the $\mathrm{Z}$ chromosome in $O$. hubbsi (Takehana et al. 2007b). In addition, a telomeric inversion around the sexdetermining locus was observed, and recombination between $\mathrm{Z}$ and $\mathrm{W}$ chromosomes around the locus was absent, suggesting that this inversion suppressed crossing over between $\mathrm{Z}$ and $\mathrm{W}$ chromosomes around the locus. In this study, we found that the $18 \mathrm{~S}-28 \mathrm{~S}$ rDNA, as well as the Bst $\mathrm{NI}$ family sequence, were located on the heterochromatin region of the $\mathrm{W}$ chromosome, indicating that the heterochromatin blocks of the $\mathrm{W}$ chromosome consist of different repetitive elements. Therefore, the accumulation of these repetitive sequences in this non-recombining region may compose the $\mathrm{W}$ chromosome-specific heterochromatin blocks and enlarge the W chromosome.

rDNA sequences shared between nonhomologous W chromosomes in Oryzias

FISH mapping demonstrated that the 18S-28S rDNA sequences were located on the $\mathrm{W}$ chromosome but not on the $\mathrm{Z}$ chromosome in both $O$. hubbsi and $O$. javanicus. Synteny of the sex-linked genes is not conserved between these species, suggesting that the rDNA sequences are shared between the nonhomologous $\mathrm{W}$ chromosomes. We propose two alternative scenarios for this situation. The first is a translocation of a $\mathrm{W}$ chromosomal region. In this case, a small region including the sex-determining gene and the rDNAs would move to another chromosome, thereby producing a novel $\mathrm{W}$ chromosome. A shared sex chromosome repeat in different $\mathrm{Y}$ chromosomes was also reported in the salmonid fishes, Oncorhynchus kisutch and Oncorhynchus tshawytscha (Phillips et al. 2005). They have a common Y-specific marker adjacent to the sex-determining locus at the telomeric regions, although the remaining part of the chromosome does not appear to be homologous, which supports our hypothesis.

The second scenario is that the same rDNAs are independently amplified on different $\mathrm{W}$ chromosomes. Sex chromosomes bearing rDNAs/NORs have been reported in some vertebrates, such as mammals, reptiles, and amphibians (Kawai et al. 2007; O’Meally et al. 2010; Schmid et al. 1983; Wiley 2003), suggesting that the accumulation of rDNAs is a common property of sex chromosome differentiation in vertebrates. Strong signals of rDNAs/NORs were observed on $\mathrm{X}$ or $\mathrm{W}$ chromosome in these cases; therefore, the copy number of the rRNA genes was considered to be higher in females than in males, implying a functional significance for female-specific amplification of NORs. Without molecular characterization of the sex-determining region, however, it is difficult to choose an appropriate hypothesis in the case of $O$. hubbsi and $O$. javanicus. To address this issue, we have constructed bacterial artificial chromosome libraries of $O$. hubbsi and $O$. javanicus. Future sequence comparisons around the sexdetermining region between these species will unravel the origins and differentiation processes of the $\mathrm{ZW}$ sex chromosomes in these species.

Acknowledgments This work was supported in part by Grants-in-Aid for Scientific Research, 16370094 (to MS), 18017006 (to KN), and 18870007 (to YT) from the Ministry of Education, Culture, Sports, Science and Technology, Japan and the Sasakawa Scientific Research Grant (to YT) from The Japan Science Society.

\section{References}

Charlesworth D, Charlesworth B, Marais G (2005) Steps in the evolution of heteromorphic sex chromosomes. Heredity 95:118-128

Huang X, Wang J, Aluru S, Yang SP, Hillier L (2003) PCAP: a whole-genome assembly program. Genome Res 13:21642170

Kawai A, Nishida-Umehara C, Ishijima J, Tsuda Y, Ota H, Matsuda Y (2007) Different origins of bird and reptile sex chromosomes inferred from comparative mapping of chicken Z-linked genes. Cytogenet Genome Res 117:92102 
Kondo M, Nanda I, Hornung U, Schmid M, Schartl M (2004) Evolutionary origin of the medaka Y chromosome. Curr Biol 14:1664-1669

Matsubara K, Tarui H, Toriba M et al (2006) Evidence for different origin of sex chromosomes in snakes, birds, and mammals and step-wise differentiation of snake sex chromosomes. Proc Natl Acad Sci U S A 103:1819018195

Matsuda Y, Chapman VM (1995) Application of fluorescence in situ hybridization in genome analysis of the mouse. Electrophoresis 16:261-272

Matsuda M, Matsuda C, Hamaguchi S, Sakaizumi M (1998) Identification of the sex chromosomes of the medaka, Oryzias latipes, by fluorescence in situ hybridization. Cytogenet Cell Genet 82:257-262

Nanda I, Shan Z, Schartl M et al (1999) 300 million years of conserved synteny between chicken $\mathrm{Z}$ and human chromosome 9. Nat Genet 21:258-259

O'Meally D, Patel HR, Stiglec R et al (2010) Non-homologous sex chromosomes of birds and snakes share repetitive sequences. Chromosome Res 18:787-800

Ohno S (1967) Sex chromosomes and sex-linked genes. Springer, Berlin

Phillips RB, Morasch MR, Park LK, Naish KA, Devlin RH (2005) Identification of the sex chromosome pair in coho salmon (Oncorhynchus kisutch): lack of conservation of the sex linkage group with chinook salmon (Oncorhynchus tshawytscha). Cytogenet Genome Res 111:166-170

Schmid M, Haaf T, Geile B, Sims S (1983) Chromosome banding in Amphibia. VIII. An unusual XY/XX-sex chromosome system in Gastrotheca riobambae (Anura, Hylidae). Chromosoma 88:69-82

Singer MF (1982) Highly repeated sequences in mammalian genomes. Int Rev Cytol 76:67-112
Sonnhammer ELL, Durbin R (1995) A dot-matrix program with dynamic threshold control suited for genomic DNA and protein sequence analysis. Gene 167:GC1-GC10

Takehana Y, Naruse K, Sakaizumi M (2005) Molecular phylogeny of the medaka fishes genus Oryzias (Beloniformes: Adrianichthyidae) based on nuclear and mitochondrial DNA sequences. Mol Phylogenet Evol 36:417-428

Takehana Y, Demiyah D, Naruse K, Hamaguchi S, Sakaizumi M (2007a) Evolution of different Y chromosomes in two medaka species, Oryzias dancena and O. latipes. Genetics 175:1335-1340

Takehana Y, Naruse K, Hamaguchi S, Sakaizumi M (2007b) Evolution of $\mathrm{ZZ/ZW}$ and $\mathrm{XX} / \mathrm{XY}$ sex-determination systems in the closely related medaka species, Oryzias hubbsi and $O$. dancena. Chromosoma 116:463-470

Takehana Y, Hamaguchi S, Sakaizumi M (2008) Different origins of ZZ/ZW sex chromosomes in closely related medaka fishes, Oryzias javanicus and O. hubbsi. Chromosome Res 16:801-811

Tanaka K, Takehana Y, Naruse K, Hamaguchi S, Sakaizumi M (2007) Evidence for different origins of sex chromosomes in closely related medaka fishes: substitution of the master sex-determining gene. Genetics 177:2075-2081

Uwa H (1986) Karyotype evolution and geographical distribution in the ricefish, genus Oryzias (Oryziidae). In: Uyeno T, Arai R, Taniuchi T, Matsuura K (eds) Indo-Pacific fish biology: Proceedings of the Second International Conference on Indo-Pacific Fishes. Ichthyological Society of Japan, Tokyo, pp 867-876

Uwa H, Iwata A (1981) Karyotype and cellular DNA content of Oryzias javanicus (Oryziatidae, Pisces). Chromosome Inf Serv 31:24-26

Wiley JE (2003) Replication banding and FISH analysis reveal the origin of the Hyla femoralis karyotype and XY/XX sex chromosomes. Cytogenet Genome Res 101:80-83 